\title{
Article
}

\section{Regulation of Krüppel-like Factor 15 Expression by Herpes Simplex Virus Type 1 or Bovine Herpesvirus 1 Productive Infection.}

\author{
Fouad S. El-mayet ${ }^{1,2}$, Kelly S. Harrison ${ }^{1}$ and Clinton Jones ${ }^{1^{*}}$ \\ 1 Oklahoma State University, College of Veterinary Medicine, Department of Veterinary Pathobiology, \\ Stillwater, OK \\ 2 Benha University, Faculty of Veterinary Medicine, Department of Virology, Moshtohor 13736, Kaliobyia, \\ Egypt \\ * Correspondence: author: clint.jones10@okstate.edu; Tel.: 405-744-1842
}

\begin{abstract}
Expression of Krüppel-like factor 15 (KLF15), a stress induced transcription factor, is induced during bovine herpesvirus 1 (BoHV-1) reactivation from latency, and KLF15 stimulates BoHV-1 replication. Transient transfection studies revealed KLF15 and glucocorticoid receptor (GR) cooperatively transactivate the BoHV-1 immediate early transcription unit 1 (IEtu1), herpes simplex virus type 1 (HSV-1) infected cell protein 0 (ICP0), and ICP4 promoter. The IEtu1 promoter drives expression of $\mathrm{bICP} 0$ and bICP4, two key BoHV-1 transcriptional regulatory proteins. Based on these studies, we hypothesized infection is a stressful stimulus that increases KLF15 expression and enhances productive infection. New studies demonstrated that silencing KLF15 impaired HSV-1 productive infection and KLF15 steady state protein levels were increased at late stages of productive infection. KLF15 was primarily localized to the nucleus following infection of cultured cells with HSV-1, but not BoHV-1. When cells were transfected with a KLF15 promoter construct and then infected with HSV-1, promoter activity was significantly increased. The ICP0 gene and to a lesser extent bICP0 transactivated the KLF15 promoter in the absence of other viral proteins. In contrast, BoHV-1 or HSV-1 encoded VP16 had no effect on KLF15 promoter activity. Collectively, these studies revealed HSV-1 and BoHV-1 productive infection increased KLF15 steady state protein levels, which correlated with increased virus production.
\end{abstract}

Keywords: herpes simplex virus type 1 (HSV-1), bovine herpesvirus 1 (BoHV-1), Krüppel-like factor 15 (KLF15), infected cell protein 0 (ICP0), and BoHV-1 ICP0 (bICP0)

\section{Introduction}

Bovine herpesvirus 1 (BoHV-1) and herpes simplex virus type 1 (HSV-1) are important pathogens of their respective hosts (1). Productive infection leads to high levels of virus production and viral transmission. Viral genes are expressed in three distinct phases: immediate early (IE), early, and late (2,3). A tegument protein (VP16) specifically activates IE promoters by interacting with two cellular proteins, Oct-1 and HCF-1 (46). Infected cell protein 0 (ICP0) and ICP4 are key viral transcriptional regulatory proteins that drive early and late gene expression. Three BoHV-1 immediate early (IE) genes express mRNA translated into infected cell protein 0 (bICP0), bICP4, and bICP22 $(7,8)$. While expression of HSV-1 encoded ICP0 and ICP4 is regulated by separate promoters, the BoHV-1 immediate early transcription unit 1 (IEtu1) promoter controls IE expression of a single mRNA that is alternatively spliced to produce bICP0 and bICP4 proteins (7-9). A separate bICP0 E promoter sustains bICP0 expression throughout productive infection (9). During productive infection, BoHV-1 and HSV-1 encode early and late genes that generally have similar functions.

Following acute infection of mucosal surfaces, BoHV-1 and HSV-1 establish a lifelong latent infection in neurons (10-12). In contrast to productive infection, viral gene 
expression in latently infected neurons is restricted to the BoHV-1 encoded latency related (LR) gene or HSV-1 latency associated transcript (LAT). These genes encode multiple products that promote neuronal survival by inhibiting apoptosis and expression of viral regulatory genes important for productive infection (13-16). The LR gene encodes a protein (ORF2) that inhibits apoptosis (13) whereas LAT appears to be a non-protein coding locus, reviewed in (17). LR gene products and LAT are predicted to promote reactivation from latency by maintaining a pool of latently infected neurons that can successfully reactivate from latency. Stress, in general, increases the frequency of BoHV-1 $(11,18)$ and HSV-1 $(19,20)$ reactivation from latency. The synthetic corticosteroid dexamethasone (DEX) triggers BoHV-1 reactivation from latency, reviewed in (21), and accelerates HSV1 explant induced reactivation $(22,23)$. BoHV-1 $(24,25)$ and $\operatorname{HSV}-1(26,27)$ productive infection are also stimulated by DEX. Corticosteroids bind and activate the glucocorticoid receptor (GR) and mineralocorticoid receptor (MR) (28) suggesting these nuclear hormone receptors directly stimulate reactivation from latency.

Cellular transcription factors were identified in TG neurons within the first three hours following DEX treatment of BoHV-1 latently infected calves (29). Several members of the Krüppel-like factor (KLF) family of transcription factors (30) were identified in this study, including KLF15 (29). Additionally, explant of mouse TG contain more TG neurons that express KLF15 if the tissue is incubated with DEX (31). Furthermore, the HSV1 ICP0 promoter is cooperatively transactivated by GR and KLF15 and productive infection is impaired when cells are treated with a GR-specific antagonist (27). Since GR and KLF15 stimulate BoHV-1 productive infection (25) and transactivates key viral promoters, stressful stimuli can trigger reactivation from latency.

The primary objectives of this study were to test what effect BoHV-1 and HSV-1 had on KLF15 expression and whether KLF15 mediates productive infection. Additional studies examined the effect viral infection has on KLF15 subcellular localization and whether viral genes transactivated the KLF15 promoter activity.

\section{Materials and Methods}

Cells and Viruses

Murine neuroblastoma (Neuro-2A), Human neuroblastoma (SH-SY5Y) and Vero cells were grown in Minimal Essential Media (MEM) supplemented with $5 \%$ fetal bovine serum (FBS). Madin-Darby bovine kidney cells (MDBK) were grown in MEM supplemented with $10 \%$ FBS. All media contained penicillin $(10 \mathrm{U} / \mathrm{ml})$ and streptomycin $(100$ $\mu \mathrm{g} / \mathrm{ml})$.

The BoHV-1 Cooper strain (wt virus) is the North American prototype strain and was obtained from the National Veterinary Services Laboratory, Animal and Plant Health Inspection Services, Ames, Iowa. Stock cultures of BoHV-1 were prepared in CRIB or MDBK cells. The HSV-1 McKrae strain was obtained from the late Dr. Steven Wechsler (University of California, Irvine) and stock cultures prepared in Vero cells.

\section{Plasmids}

A 4.3-kb fragment of the human KLF15 promoter upstream of the ATG start codon was subcloned into the KpnI/EcoRV cloning sites of the pGL4.20 firefly luciferase reporter plasmid (Promega, Madison, WI) to generate pGL4.20-hKLF15 (32). The bICP0 expression construct was obtained from M Schwytzer (Zürich, Switzerland). The HSV-1 ICP0 expression construct was obtained from P. Schaffer (Harvard University). The VP16 open reading frames for BoHV-1 and HSV-1 were synthesized by GenScript and cloned into a Flag-tagged vector such that these constructs express a Flag-tagged VP16 protein.

SDS-polyacrylamide gels and Western blots

The designated cultured cells were infected with wt HSV-1 or BoHV-1 at a multiplicity of infection (MOI) of 1 PFU/cell and cell lysate collected at various times (hours) after 
infection. Cultures were washed with PBS and suspended in RIPA lysis buffer (50 mM Tris HCL (pH 8.0), 150 mM NaCL, 2 mM EDTA (pH 8.0), 1\% NP-40, 0.5\% sodium deoxycholate, $0.1 \%$ SDS) and protease inhibitor (Roche Molecular Biochemicals). Cell lysate was incubated on ice for $30 \mathrm{~min}$, sonicated, and then clarified by centrifugation at 15,000 $\mathrm{xg}$ at $4^{\circ} \mathrm{C}$ for $15 \mathrm{~min}$. Protein concentrations were quantified by the Bradford assay (BioRad). For SDS-PAGE, proteins were mixed with an equal amount of $2 X$ sample loading buffer (62.5 mM Tris- $\mathrm{HCl}$ [pH 6.8], 2\% SDS, $50 \mathrm{mM}$ dithiothreitol, 0.1\% bromophenol blue, $10 \%$ glycerol) and boiled for $5 \mathrm{~min}$. Proteins were separated in 10\% SDS-PAGE gels. After electrophoresis, proteins were transferred onto a polyvinylidene difluoride membrane (Immobilon-P; Millipore) and blocked for $1 \mathrm{~h}$ in 5\% w/v nonfat dry milk with $1 \times$ Tris-buffered saline- $0.1 \%$ Tween 20 (TBS-T). Membranes were incubated with the designated primary antibody at $4^{\circ} \mathrm{C}$ with gentle shaking overnight. The primary antibody was diluted 1:1,000 in blocking solution. Antibodies directed against $\beta$-tubulin (Fisher Scientific; catalogue \# MA5-16308) and Glyceraldehyde-3-Phosphate Dehydrogenase (GAPDH; Santa Cruz Biotechnology, Santa Cruz, CA) was used as a loading control. The KLF15 primary antibody (ab167192) and ICP4 antibody were purchased from Abcam (ab6514). A peptide-specific rabbit antibody directed against bICP4 was produced in rabbits by Affinity Bioreagents (Golden, CO). The secondary donkey anti-rabbit antibody (NA9340V) was purchased from GE Healthcare and secondary sheep anti-mouse antibody was purchased from GE Healthcare. After 45 min of washing with TBS-T, blots were incubated with secondary antibodies (peroxidase-conjugated immunoglobulin $G$ (Amersham Biosciences), which was diluted 1: 2,000 in 5\% nonfat milk in TBS-T for $1 \mathrm{~h}$. Blots were washed 45 min with TBS-T, exposed to Amersham ECL reagents, and imaged using an Amersham imager 600 (GE).

\section{Nuclear and cytoplasmic fractionation}

Vero or MDBK cells were infected with wt HSV-1 or BoHV-1 at a MOI of 1 PFU/cell for 8,16 or 24 hours after infection. The ReadyPrep ${ }^{\mathrm{TM}}$ Protein Extraction Kit (Cytoplasmic/Nuclear) was purchased from Bio-Rad, USA (catalogue \# 163-2089) and used to separate cytoplasmic proteins from nuclear proteins. Cells were harvested by centrifugation at 2,500 x $\mathrm{g}$ for 5 minutes and washed twice in cold phosphate buffered saline (PBS). Cells were suspended in ice-cold cytoplasmic protein extraction buffer (CPEB) with Protease inhibitors and incubated on ice for $30 \mathrm{~min}$. Cell suspensions were gently passed through 20-gauge needle (10-20 strokes) to lyse cells without damaging nuclei. Cell lysate was centrifuged at $1,000 \times \mathrm{g}$ for 10 minutes at $4^{\circ} \mathrm{C}$. Supernatant containing cytoplasmic proteins was immediately transferred to a new tube (on ice). The remaining nuclear pellet was washed one time with CPEB, and the supernatant discarded. The nuclear pellet was suspended in freshly prepared protein solubilization buffer (PSB) and vortexed 45 times, $60 \mathrm{sec}$ each, to solubilize the nuclear proteins. Centrifugation $(16,000 \mathrm{x} \mathrm{g})$ was performed for 15-20 minutes at room temperature to pellet genomic DNA and other debris. The clarified supernatant was transferred into a new microcentrifuge tube labeled Nuclear Protein Fraction. Protein concentrations were quantified by the Bradford assay and standard 10\% SDS-polyacrylamide gels were used to analyze KLF15 protein levels. An antibody directed against histone H3 (Abcam; ab1191) was diluted 1:500 and used as a control for fractionation studies.

\section{Immunofluorescence}

Vero or MDBK cells seeded into 2-well chamber slides (Nunc. Inc, IL, USA) were incubated in $\mathrm{MEM}$ supplemented with $10 \% \mathrm{FBS}$ at $37^{\circ} \mathrm{C}, 5 \% \mathrm{CO} 2$ for 24 hours. Cells were mock infected or infected with HSV-1 or BoHV-1 at an MOI of 1 for 16 hours. Cells were fixed in 4\% paraformaldehyde in PBS pH 7.4 for $10 \mathrm{~min}$ at room temperature, and permeabilized with $0.25 \%$ Triton X-100 in PBS pH 7.4 for 10 min at room temperature, blocked with 1\% BSA in PBST (PBS+ 0.1\% Tween 20) for $30 \mathrm{~min}$, and incubated with anti-KLF15 
antibody (Abcam, ab167192, at concentration of $10 \mu \mathrm{g} / \mathrm{ml}$ ) in 1\% BSA in PBST for $12 \mathrm{~h}$. After three washes, cells were incubated with Alexa Fluor 488 goat anti-mouse IgG $(\mathrm{H}+\mathrm{L})$ (Invitrogen, A-11001, 1:500 dilution) for $1 \mathrm{~h}$ in the dark. After three washes, DAPI (4',6diamidino-2-phenylindole) staining was performed to visualize the nucleus. Slides were covered with coverslips by using Gel Mount aqueous mounting medium (Electron Microscopy Sciences). Images were obtained by confocal microscopy (Leica).

\section{Transfection and dual-luciferase reporter assays}

For productive infection assays, Vero cells were transfected with a reporter construct containing the human KLF15 promoter (pGL4.20-hKLF15) obtained kindly from Dr. Yiqing Guo (Stony Brook University, New York). Lipofectamine 3000 (catalog no. L3000075; Invitrogen) was used according to the manufacturer's instructions. An empty vector was added as needed. Twenty-four hours later, cells were infected with BoHV-1 or HSV-1 at an MOI of 0.1 or 0.5 for 24 hours. Cells were harvested and protein lysate subjected to a dual-luciferase assay using a commercially available kit (E1910; Promega). Luminescence was measured using a GloMax 20/20 luminometer (E5331; Promega). Data for luciferase activity were averaged from the results of multiple transfections experiments (at least three independent experiments were performed).

\section{Analysis of KLF15 and scrambled siRNA on cell viability and productive infection}

A mouse specific KLF15 siRNA (SR413764, Locus ID 66277) was purchased from Origene technologies (Rockville, MD). A universal scrambled negative control siRNA was included (SR30004). Both siRNAs were reconstituted in RNAse-free suspension buffer. Neuro-2A cells were grown in MEM containing 2\% charcoal stripped FBS and transfected with increasing concentrations of siRNA duplexes using Lipofectamine 3000 according to manufacturer instructions. Transfections were incubated at $37^{\circ} \mathrm{C}, 5 \% \mathrm{CO} 2$ for $48 \mathrm{~h}$ prior to trypan blue exclusion assay using the BioRad TC20 automated cell counter.

Neuro-2A cells were grown as above and transfected with increasing concentrations of KLF15 siRNA duplexes using Lipofectamine 3000 . The universal scrambled negative control siRNA $(25 \mu \mathrm{M})$ was included as a control. Cells were then infected with HSV-1 at an MOI of 1 for $1 \mathrm{~h}$ at $37^{\circ} \mathrm{C}, 5 \% \mathrm{CO} 2$ with rocking. Media was replaced and infections incubated for 24 hours. Media and cells were harvested, freeze-thawed at $-80 / 37^{\circ} \mathrm{C}$ three times prior to total virus enumeration using plaque assay as described previously $(27,33)$.

\section{Results}

Silencing KLF15 significantly reduced HSV-1 productive infection

Previous studies demonstrated that cotransfecting GR and KLF15 with BoHV-1 genomic DNA stimulated productive infection more efficiently than KLF15 or GR alone (25). However, the effects of KLF15 on HSV-1 productive infection had not been examined. Consequently, we examined the effect of silencing KLF15 on productively infected Neuro2A cells because they are neuronal cells that can be differentiated into dopaminergic-like neurons (34). Neuro-2A cells can be readily transfected whereas human neuronal cells are not readily transfected. Initial studies tested whether the KLF15 siRNA and negative control siRNA influenced cell toxicity. The KLF15 siRNA did not significantly increase cell toxicity of Neuro-2A cells at concentrations up to $50 \mathrm{nM}$ (Figure 1A). Furthermore control siRNA did not significantly reduce cell viability up to concentrations of $50 \mathrm{~nm}$ (Figure 1). Western blot analysis demonstrated that the KLF15 siRNA reduced steady state levels via a dose-dependent response (data not shown).

Subsequent studies analyzed the effect of HSV-1 infection when the KLF15 siRNA was transfected into Neuro-2A cells and then cells infected with HSV-1 24 hours later. At 24 hours after infection, the amount of virus in cultured cells was quantified by plaque assays. These studies revealed that when Neuro-2A cells were transfected with 10 and 25 nM KLF15 siRNA there was a signficant reduction of HSV-1 virus production (Figure 
1C). When cultures were transfected with $50 \mathrm{nM}$ of the KLF15 siRNA and then infected with HSV-1, there were no detectable plaques at 24 hours after infection. In summary, these studies suggested that KLF15 was important for efficient HSV-1 replication in Neuro-2A cells.
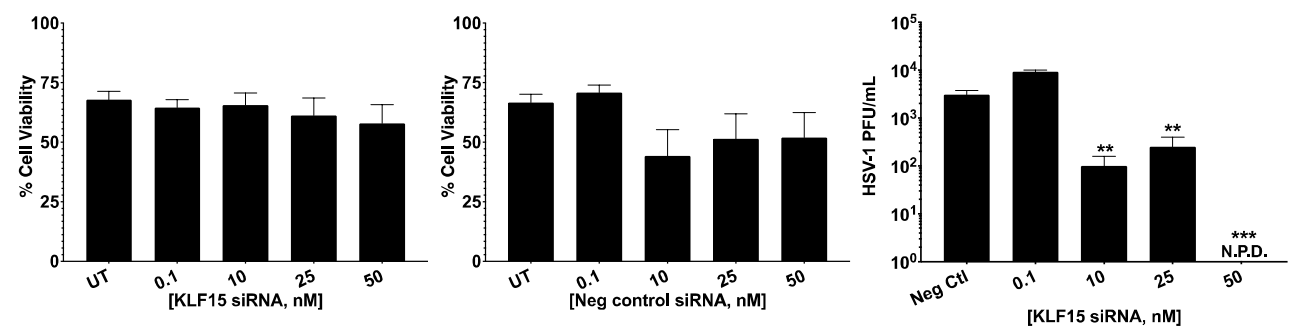

Figure 1. KLF15 siRNA reduces HSV-1 infection in Neuro-2A cells. KLF15 (Panel A) or the universal scrambled negative control (Panel B) were transfected into Neuro-2A cells at the designated concentrations of siRNA using Lipofectamine 3000 . Transfections were incubated at $37^{\circ} \mathrm{C}$, $5 \% \mathrm{CO} 2$ for $48 \mathrm{~h}$ prior to trypan blue exclusion assay using the BioRad TC20 automated cell counter. Data are shown as mean +SEM for triplicate wells of triplicate experiments. UT: untransfected. Panel C: KLF15 siRNA reduces HSV-1 productive infection. Neuro-2a cells were grown in MEM containing $2 \%$ stripped FBS and transfected with increasing concentrations of KLF15 siRNA duplexes using Lipofectamine 3000 according to manufacturer instructions at $37^{\circ} \mathrm{C}, 5 \% \mathrm{CO} 2$ for $24 \mathrm{~h}$. Cells were then infected with $\mathrm{HSV}-1$ at a MOI of 1 for $1 \mathrm{~h}$ at $37^{\circ} \mathrm{C}, 5 \%$ $\mathrm{CO} 2$ with rocking. Media was replaced and infected cells incubated for $24 \mathrm{~h}$. Virus in the designated cultures was measured by plaque assays. Data are shown as mean +SEM for duplicate wells of triplicate experiments. N.P. D.: No plaques were detected; ${ }^{* *} \mathrm{p}<0.005 ;{ }^{* * *} \mathrm{p}<0.001$ by student's T-test.

\section{KLF15 steady state protein levels increase following HSV-1 infection}

To better understand the relationship between KLF15 and HSV-1 productive infection, KLF15 steady state protein levels were examined following infection of permissive cells with HSV-1. Vero (Figure 2A) or human neuroblastoma (SH-SY5Y) cells (Figure 2B) were infected with HSV-1 and whole cell lysate collected at the designated times after infection. Western blot analysis indicated KLF15 steady state protein levels increased as a function of time after infection of Vero cells (Figure 2A). For example, there was an approximate 2 -fold increase by $8 \mathrm{~h}$ after infection. Furthermore, there was at least a 3-fold increase at 16 and $24 \mathrm{~h}$ after infection of Vero cells, which was significantly different relative to Vero cells mock infected for 2 or 24 hours (Figure 2C). At $16 \mathrm{~h}$ and $24 \mathrm{~h}$ after infection of SH-SY5Y cells, there was also a signficant increase in KLF15 steady state protein levels when compared to cells mock infected for 2 or 24 hours (Figure 2B and D). As expected, ICP4 expression was readily detected by 4 hours after infection and increased as viral replication increased. In contrast to KLF15, KLF4 protein levels were not increased following infection of Vero or SH-SY5Y (data not shown). 

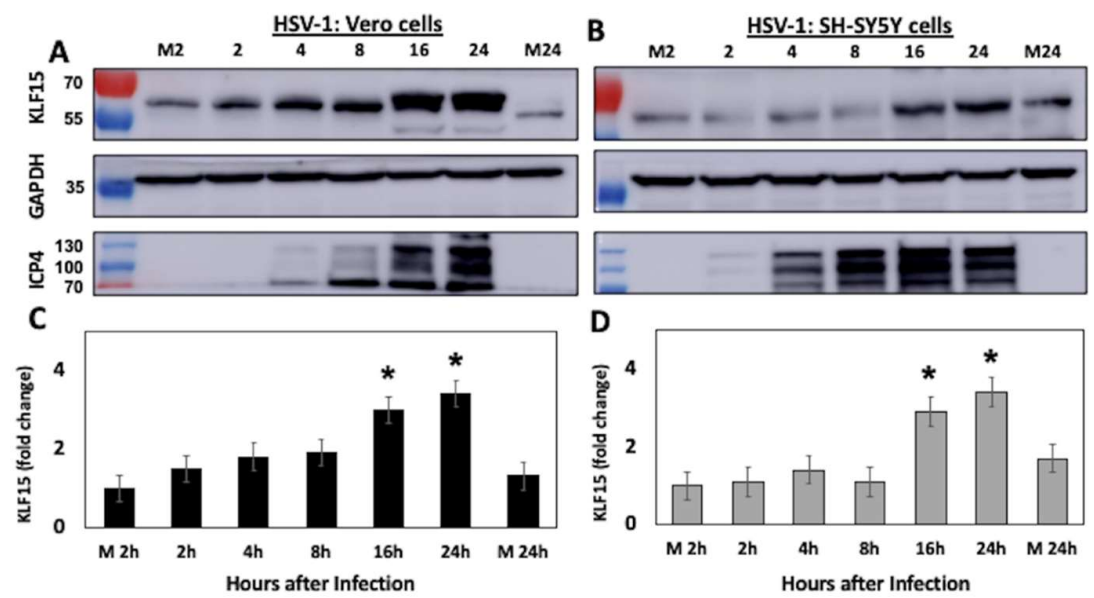

Figure 2. Western blot analysis of KLF15 protein levels during HSV-1 productive infection. Vero (Panel A) or human neuroblastoma (SH-SY5Y) cells (Panel B) were mock infected or infected with HSV-1 (MOI = 1 PFU/cell). At the designated times after infection (hours), cells were collected and lysed with RIPA buffer. Proteins were separated by SDS-PAGE, and Western blot analysis performed using the KLF15 polyclonal antibody $(1 \mu \mathrm{g} / \mathrm{ml})$. As a loading control, GAPDH levels were examined. For each lane, $50 \mu \mathrm{g}$ protein was loaded. Lanes M2 or M24 are 2 or $24 \mathrm{~h}$ after mock infection. A representative blot of three independent experiments is shown. Size of molecular weight markers are shown on the left of the blot. Panels C and D show the quantification of KLF15 by densitometry $(n=3)$ using ImageJ $1.26 \mathrm{t}$ software (National Institutes of Health; rsb.info.nih.gov/ij) and error bars denote the standard error of the mean (SEM). Significant differences $(\mathrm{P}<0.05)$ between cells versus M2 or M24 (students T test) are denoted by an asterisk $(\mathrm{p}<0.05)$.

Immunofluorescence was performed to compare KLF15 protein expression in infected Vero cells at 16 hours after infection versus mock infected cells (Figure 3). Relative to mock infected cells (Figure 3A), KLF15 protein expression was readily detected at 16 hours of infection (Figure 3B). Collectively, these studies indicated HSV-1 productive infection increased KLF15 steady state protein levels at late times after infection of Vero and SH-SY5Y cells.
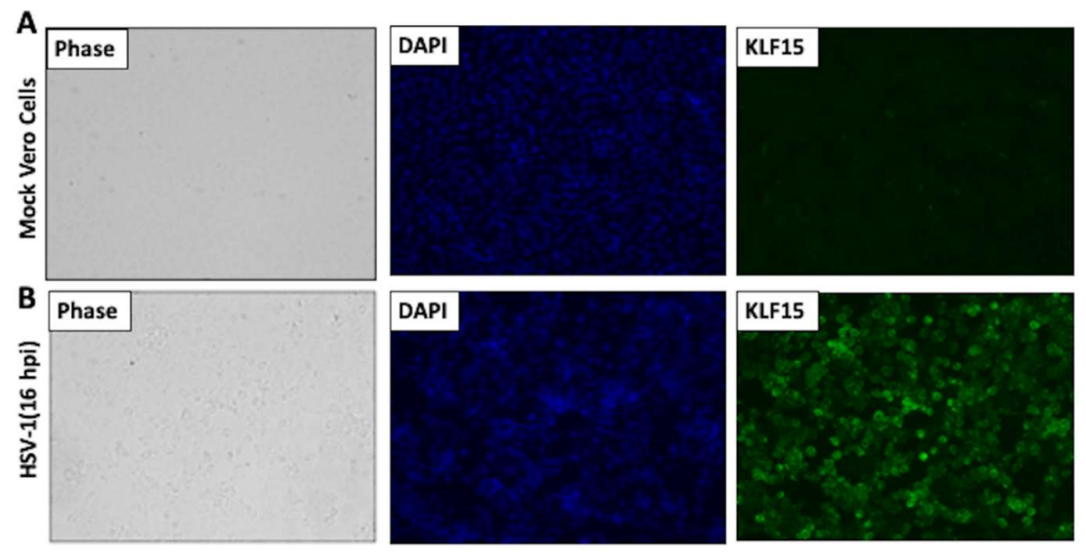

Figure 3. Immunofluorescence studies demonstrated KLF15 protein levels are increased during HSV-1 productive infection. Vero cells were seeded in chamber slides for 24 hours and then mock infected (Panel A) or infected (Panel B) with HSV-1 (MOI of 1) for $16 \mathrm{~h}$. After washing with PBS for three times, cells were fixed with $4 \%$ paraformaldehyde in PBS and KLF15 detected by IFA using the KLF15 specific antibody (green). DAPI staining was used to detect nuclear DNA (blue). Images were obtained by performing confocal microscopy (Leica). These images are representative of three independent experiments. 


\section{KLF15 steady state protein levels increase following BoHV-1 infection}

Bovine kidney (MDBK) or mouse neuroblastoma (Neuro-2A) cells were infected with BoHV-1 and whole cell lysate collected at the designated times after infection. Western blot studies revealed significantly higher KLF15 steady state protein levels at 16 and 24 hours after infection (Figure 4A and C). The results in MDBK cells were compared to Neuro-2A cells because BoHV-1 does not replicate at high levels in Neuro-2A cells (35). In contrast to MDBK cells, KLF15 steady state protein levels (Figure 4C) were not significantly higher after infection (Figure 4D). When compared to mock infected cells (Figure 5A), immunostaining confirmed an increase in KLF15 expression in infected MDBK cells (Figure 5B), which was consistent with Western blot studies (Figure 4A). In summary, these studies demonstrated there is a correlation between efficient BoHV-1 productive infection and increased KLF15 protein steady state levels at late times after infection.

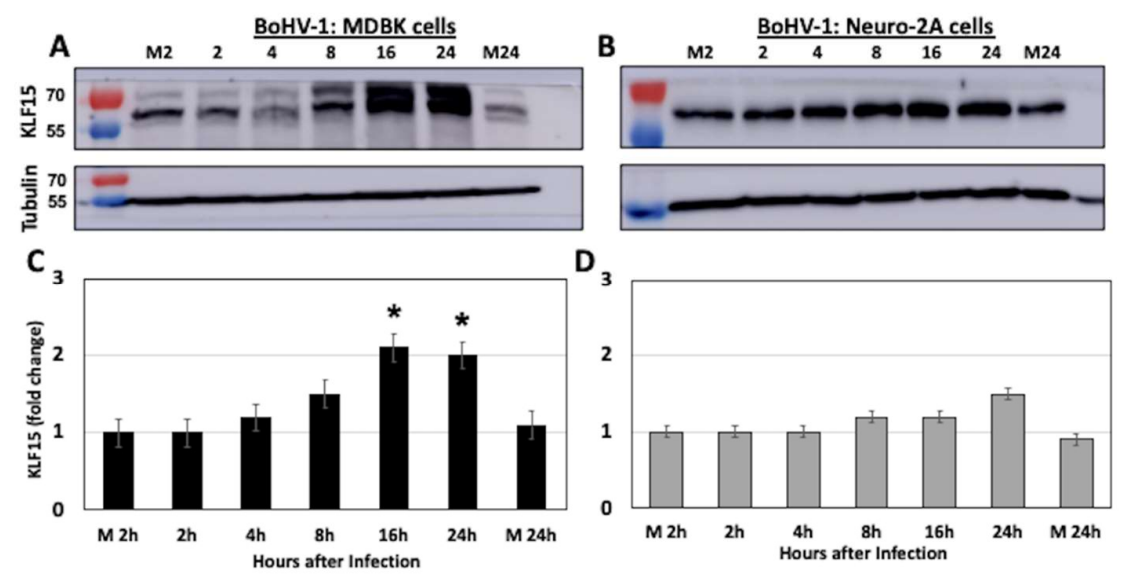

Figure 4. Western blot analysis of KLF15 protein levels during BoHV-1 productive infection. MDBK cells (Panel A) or Neuro-2A cells (Panel B) were mock infected or infected with BoHV-1 $(\mathrm{MOI}=1 \mathrm{PFU} / \mathrm{cell})$. At the designated times after infection (hours), cells were collected, proteins separated by SDS-PAGE, and Western blot analysis performed using the KLF15 polyclonal antibody $(1 \mu \mathrm{g} / \mathrm{ml})$. As a loading control, tubulin levels were examined. For each lane, $50 \mu \mathrm{g}$ protein was loaded. Lanes M2 or M24 are 2 or $24 \mathrm{~h}$ respectively after mock infection. Position of molecular weight markers are shown to the left of the Western Blot. Panels C and D summarize the quantification of KLF15 by densitometry $(n=3)$ using ImageJ $1.26 t$ software (National Institutes of Health; rsb.info.nih.gov/ij) and error bars denote the standard error of the mean (SEM). Significant differences $(\mathrm{P}<0.05)$ between cells versus M2 or M24 (students T test) are denoted by an asterisk $(\mathrm{P}<0.05)$.
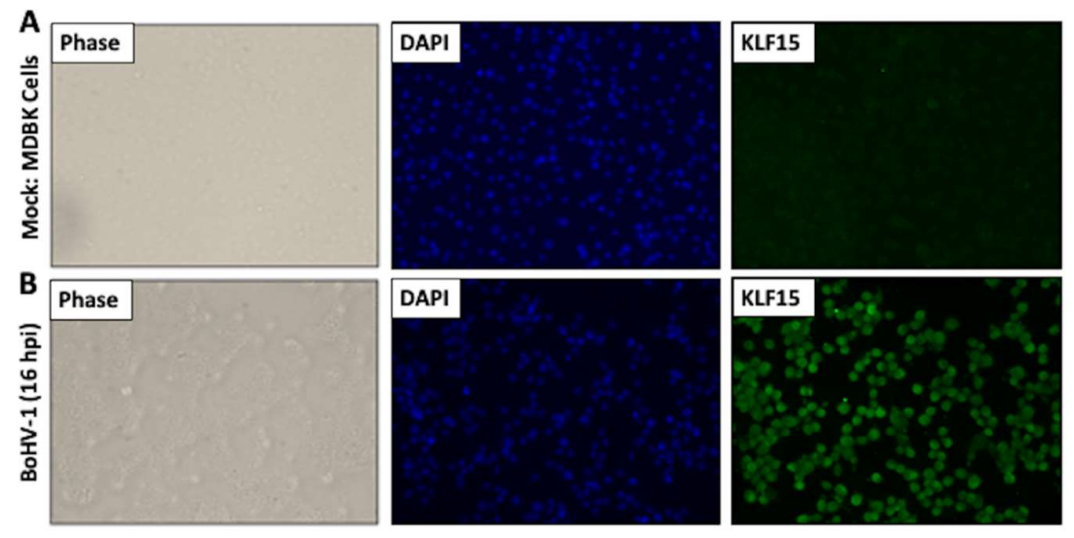

Figure 5. immunofluorescence studies confirmed KLF15 protein levels increased during BoHV1 productive infection. MDBK cells were seeded in chamber slides for 24 hours and then mock infected (Panel A) or infected (Panel B) with BoHV-1 at a MOI of 1 for $16 \mathrm{~h}$. After washing with 
PBS three times, cells were fixed with 4\% paraformaldehyde in PBS and KLF15 detected by IFA using the KLF15 antibody (green). DAPI staining was used to detect nuclear DNA (blue). Images were obtained by performing confocal microscopy (Leica). These images are representative of three independent experiments.

\section{Analysis of KLF15 localization following infection}

To test whether KLF15 is localized in the nucleus following productive infection with HSV-1 and /or BoHV-1, biochemical fractionation studies were conducted in Vero and MDBK cells at 8,16 , and 24 hours after infection. These cell lines were used because they are permissive for the respective viruses. The KLF15 protein was primarily detected in nuclear extracts of Vero cells infected for 8, 16 or $24 \mathrm{~h}$ after HSV-1 infection (Figure 6A). Conversely, KLF15 was detected in the nuclear and cytoplasmic extract following BoHV1 infection at 16 and 24 hours (Figure 6B). In fact, KLF15 was primarily detected in the cytoplasmic extract 24 hours after infection with BoHV-1. However, KLF15 was primarily detected in the nucleus of MDBK infected cells 8 hours after BoHV-1 infection. As expected, ICP4 (Panel A) and bICP4 (panel B) were primarily detected in the nucleus following infection. To ensure biochemical fractionation did not lyse nuclei of infected cells, histone $\mathrm{H} 3$ localization was examined. Histone $\mathrm{H} 3$ was detected only in the nucleus after infection with HSV-1 (Figure 6A) or BoHV-1 (Figure 6B), which was expected. In summary, this study revealed KLF15 accumulated in the nucleus of HSV-1 infected cells: conversely, KLF15 primarily localized to the cytoplasm and to a lesser extent in the nucleus of MDBK cells at 16 and 24 hours after BoHV-1 infection.

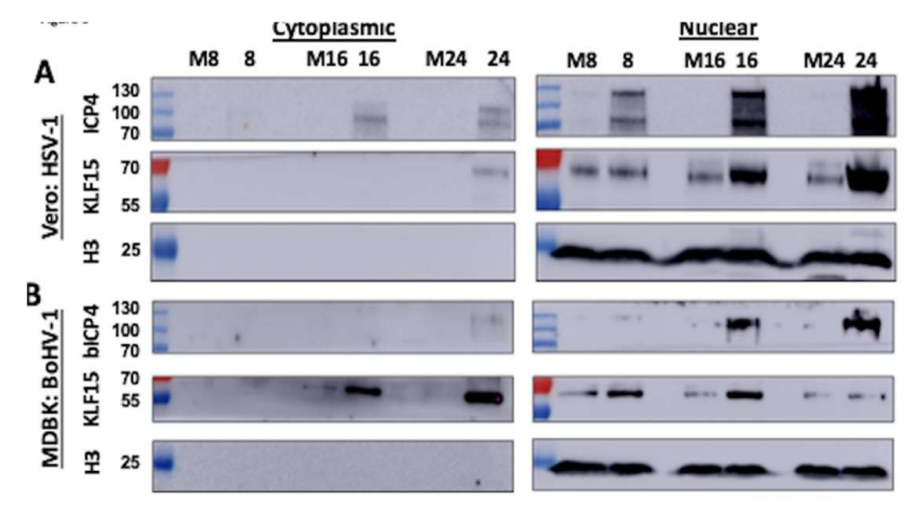

Figure 6. Localization of KLF15 after infection of permissive cells with HSV-1 or BoHV-1. Panel A: Vero cells were mock infected or infected with HSV-1 at a MOI of 1 for 8, 16, or $24 \mathrm{~h}$. Cells were harvested, and cell fractionation conducted as described in the materials and methods section. Cytoplasmic and nuclear fractions $(50 \mu \mathrm{g})$ were analyzed by Western blot using the KLF15 or ICP4 antibody $(1 \mu \mathrm{g} / \mathrm{ml})$. As a control for nuclear proteins, the respective fractions were probed with an antibody directed against Histone H3, which was diluted 1:500. Panel B: MDBK cells were infected with BoHV-1 at a MOI of 1 for 8, 16, or $24 \mathrm{~h}$. Cellular fractions were analyzed as described in panel A using the KLF15 or bICP4 antibody. Lane M is cell lysate derived from mock-infected cells: the number denotes the time after mock-infection. A representative blot from two independent studies is shown.

\section{KLF15 promoter activity is stimulated by HSV-1 infection and ICP0}

Additional studies tested whether HSV-1 and certain viral transcriptional regulators stimulated KLF15 promoter activity. Vero cells were initially transfected with a luciferase reporter construct containing the human KLF15 promoter region (32). Cells were subsequently infected with HSV-1 at an MOI of 0.1 or 0.5 PFU/cell for 24 hours. HSV-1 infection stimulated KLF15 promoter activity approximately 4-fold in Vero cells, which was significantly higher than mock-infected cells (Figure 7A). Similar studies were attempted in MDBK cells (data not shown). However, the transfection efficiency of MDBK cells is very low, which made it difficult to perform this experiment. 
The studies described in Figure 7A suggest a viral encoded protein stimulated KLF15 promoter activity, which correlated with increased KLF15 steady state protein levels following infection. ICP0 and bICP0 are promiscuous transactivators (36) suggesting these viral proteins stimulate KLF15 promoter activity. To test this prediction, Vero cells were co-transfected with the KLF15 promoter construct and increasing concentrations of a plasmid that expresses ICP0 or bICP0. The HSV-1 ICP0 expression plasmid transactivated the KLF15 promoter more than 11-fold in transfected Vero cells (Figure 7B). Although the BoHV-1 bICP0 expression plasmid transactivated the KLF15 promoter approximately 4-fold, it was not as efficient as ICP0. As a comparison to ICP0 and bICP0, VP16 expression constructs were also examined. In contrast to ICP0 and bICP0, VP16 encoded by HSV-1 or BoHV-1 did not significantly transactivate the KLF15 promoter in Vero or Neuro-2A cells (Figure 7C). Collectively, these studies demonstrated ICP0 and to a lesser extent bICP0 transactivated the KLF15 promoter in the absence of other viral genes.
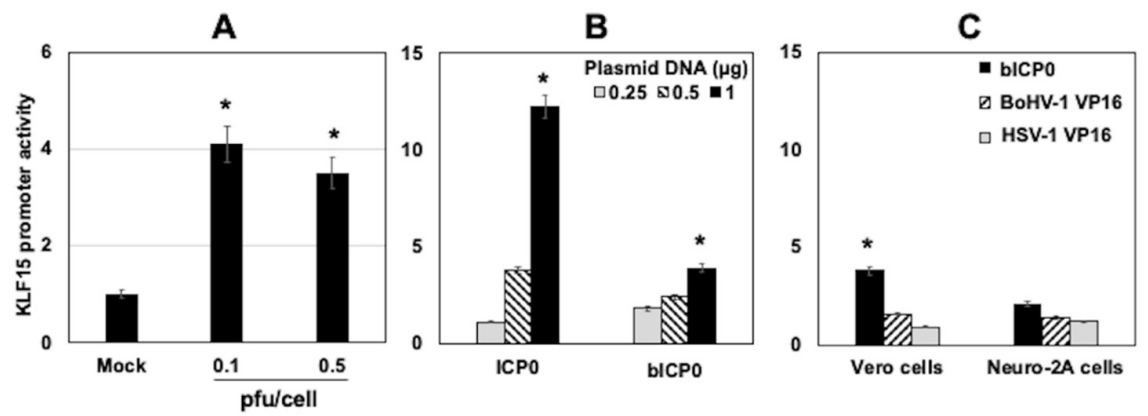

Figure 7. KLF15 promoter was activated during productive infection and ICP0. Panel A: Vero cells were transfected with $1 \mu \mathrm{g}$ of the pGL4.20-hKLF15 promoter-luciferase construct using Lipofectamine 3000. Twenty-four hours after transfection, cells were infected with HSV-1 at a MOI of 0.1 or $0.5 \mathrm{PFU} / c e l l$. At 48 hours after transfection, cells were harvested, and protein lysate subjected to a dual-luciferase assay for measuring KLF15 promoter activity. Promoter activity from mock infected cells was set at a value of 1 , and the other values were compared to mock infected cells. Panel B: Vero cells were co-transfected with $1 \mu \mathrm{g}$ of pGL4.20-hKLF15 and increasing concentrations $(0.25,0.5$ or $1 \mu \mathrm{g})$ of a plasmid expressing HSV-1 ICP0 or BoHV-1 bICP0 using Lipofectamine 3000. Panel C: Vero cells were co-transfected with $1 \mu \mathrm{g}$ of pGL4.20-hKLF15 and 1 $\mu \mathrm{g}$ of a plasmid expressing BoHV-1 bICP0, BoHV-1 VP16, or HSV-1 VP16 using Lipofectamine 3000. Basal promoter activity from cells transfected with the KLF15 promoter and empty plasmid was set at a value of 1 , and other values compared to this sample (Panels B and C). The results are the average of $n=4$ (Panel A) or $n=3$ (Panels B and C) independent experiments. Error bars denote the standard error of the mean (SEM). A significant difference $(p<0.05)$ between KLF15 promoter activity relative (students T test) is denoted by an asterisk.

\section{Discussion}

KLF family members, including KLF15, belong to the Sp1 transcription factor family $(37,38)$, and Sp1 directly activates HSV-1 gene expression because there are many Sp1 binding sites in the genome (39). The BoHV-1 genome also contains many Sp1 binding sites, suggesting Sp1 can transactivate key viral promoters. KLF family members bind GC or CA rich motifs, including certain motifs that contain consensus Sp1 binding sites $(37,40)$. Since BoHV-1 and HSV-1 are GC-rich genomes, KLF15 may stimulate numerous viral promoters. GR and KLF15 regulate gene expression dynamics and integrate signals by a feed forward transcription loop in response to stressful stimuli (41-43). The hallmark of a feed forward transcription loop is the primary factor (GR for example) stimulates KLF15 expression (44-49). Consequently, GR and KLF15 activate a novel gene expression program relative to GR or KLF15 $(41,42,50)$. Interactions between GR and KLF15 are also important for the feed forward transcription loop to be effective. Since HSV-1 infection is stimulated by corticosteroids $(26,27)$ and can increase GR and NF-kB 
steady state protein levels (26), GR and KLF15 may stimulate viral gene expression via a feed forward transcription loop in certain cell types. GR and KLF15 occupancy of ICP0 promoter sequences (27) occurred prior to ICP4 promoter sequences (51) when cultures are treated with DEX. Furthermore, GR and KLF15 occupy IEtu1 promoter sequences prior to the bICP0 E promoter (52) when cultures are treated with DEX adding support to the concept these interactions are important. Since silencing KLF15 interfered with HSV1 replication and over-expressing GR and KLF15 stimulated BoHV-1 replication (25), we predict GR and KLF15 interactions with viral promoters have biological relevance during productive infection and perhaps during stress-induced reactivation from latency.

In the absence of other viral genes, ICP0 and bICP0 transactivated KLF15 promoter activity, which correlated with increased KLF15 steady state protein levels during productive infection. ICP0 and bICP0 are promiscuous transactivators that apparently do not specifically bind DNA $(36,53)$. While bICP0 was not as effective as ICP0, it did consistently stimulate KLF15 promoter activity. Conversely HSV-1 and BoHV-1 encoded VP16 had no effect on KLF15 promoter activity. Based on the known functions of these viral proteins, we suggest two scenarios by which ICP0 and bICP0 stimulates KLF15 promoter activity. First, bICP0 and ICP0 interact with histone modifying enzymes, alter histone modification, and promote histone removal (54-57) suggesting these viral proteins remodel chromatin within the KLF15 promoter and consequently stimulate promoter activity. Secondly, the ability of bICP0 and ICP0 to function as E3 ubiquitin ligases (53, 5862) may indirectly increase KLF15 promoter activity by degrading proteins that impair KLF15 promoter activity. In the context of productive infection, we cannot rule out the possibility that other viral genes play a role in increasing KLF15 steady state protein levels.

As pointed out in the introduction, signficant differences exist in the organization of the ICP0- and ICP4-coding strategy of BoHV-1 relative to HSV-1. There are several other significant differences that exist between BoHV-1 and HSV-1. For example, the BoHV-1 IEtu1 promoter contains two consensus GREs that are essential for GR and KLF15 mediated transactivation when cultures are treated with dexamethasone $(24,25)$. Similar to the bICP0 E promoter (52), the HSV-1 ICP0 (27) and ICP4 promoter (51) do not contain consensus GREs. However, these promoters are cooperatively transactivated by GR and KLF15 suggesting KLF15, not GR, mediate transactivation of the three promoters that lack consensus GREs. For example, Sp1 sequences and KLF4 binding sites in the ICP4 promoter (51) are critical for GR and KLF15 cooperative transactivation for ICP4 enhancer sequences. Since several KLF15 binding sites have been described (63-66), merely examining the sequences of a given viral promoters may not identify a KLF15 responsive motif.

\section{Conclusions}

The studies in this report demonstrated BoHV-1 and HSV-1 stimulate KLF15 steady state protein levels during late stages of productive infection. While our studies predict KLF15 stimulates viral gene expression during productive infection, KLF15 may also enhance additional steps during late stages of productive infection. Future studies will focus on delineating the mechanism by which KLF15 regulates productive infection as well as certain aspects of reactivation from latency.

Acknowledgements: This research was supported by grants to C. Jones from the National Institute of Neurological Disorders and Stroke of the National Institutes of Health under Award Number R01NS111167, two USDA NIFA competitive grants (2016-09370 and 2018-06668), and funds from the Sitlington Endowment. Dr. Kelly Harrison is partially supported by a seed grant from the OSU College of Veterinary Medicine Research Advisory Council. The Oklahoma Center for Respiratory and Infectious Diseases (National Institutes of Health Centers for Biomedical Research Excellence Grant \#P20GM103648) also supported part of these studies. 


\section{References}

1. Jones C. 1998. Alphaherpesvirus latency: its role in disease and survival of the virus in nature. Adv Virus Res 51:81-133.

2. Honess RW, Roizman B. 1974. Regulation of herpes virus macromalecular synthesis: Cascade regulation of three groups of viral proteins. J Virol 14:8-19.

3. Honess RW, Roizman B. 1975. Regulation of herpesvirus macromolecular synthesis: sequential transition of polypeptide synthesis requires functional viral polypeptides. Proc Natl Acad Sci U S A 72:1276-80.

4. Misra V, Walker S, Hayes S, O'Hare P. 1995. The bovine herpesvirus alpha gene trans-inducing factor activates transcription by mechanisms different from those of its herpes simplex virus type 1 counterpart VP16. J Virol 69:5209-16.

5. Kristie TM. 2015. Dynamic modulation of HSV chromatin drives initiation of infection and proides targets for epigenetic therapies. Virology 479-480:555-561.

6. O'Hare P. 1993. The virion transactivator of herpes simplex virus. Seminars in Virol 4:145-155.

7. Wirth UV, Gunkel K, Engels M, Schwyzer M. 1989. Spatial and temporal distribution of bovine herpesvirus 1 transcripts. J Virol 63:4882-9.

8. Wirth UV, Vogt B, Schwyzer M. 1991. The three major immediate-early transcripts of bovine herpesvirus 1 arise from two divergent and spliced transcription units. J Virol 65:195-205.

9. Wirth UV, Fraefel C, Vogt B, Vlcek C, Paces V, Schwyzer M. 1992. Immediate-early RNA 2.9 and early RNA 2.6 of bovine herpesvirus 1 are 3 ' coterminal and encode a putative zinc finger transactivator protein. J Virol 66:2763-72.

10. Jones C. 2003. Herpes simplex virus type 1 and bovine herpesvirus 1 latency. Clin Micro Rev 16:79-95.

11. Jones C. 2013. Bovine herpes virus 1 (BHV-1) and herpes simplex virus type 1 (HSV-1) promote survival of latently infected sensory neurons, in part by inhibiting apoptosis. . J of Cell Death 6:1-16.

12. Perng G-C, Jones C. 2010. Towards an understanding of the Herpes Simplex Virus Type 1 latency-reactivation cycle. Interdisciplinary Perspectives on Infectious Diseases 2010:1-18.

13. Shen W, Jones C. 2008. Open reading frame 2, encoded by the latency-related gene of bovine herpesvirus 1 , has antiapoptotic activity in transiently transfected neuroblastoma cells. J Virol 82:10940-5.

14. Perng G-C, Jones C, Ciacci-Zanella J, Stone M, Henderson G, Yukht A, Slanina AJ, Hoffman FM, Ghiasi H, Nesburn AB, Wechsler SL. 2000. Virus-induced neuronal apoptosis blocked by the herpes simplex virus latency-associated transcript (LAT). Science 287:1500-1503.

15. Ciacci-Zanella J, Stone M, Henderson G, Jones C. 1999. The latency-related gene of bovine herpesvirus 1 inhibits programmed cell death. J Virol 73:9734-40.

16. Inman M, Perng G-C, Henderson G, Ghiasi H, Nesburn AB, Wechsler SL, Jones C. 2001. Region of herpes simplex virus type 1 latency-associated transcript sufficient for wild-type spontaneous reactivation promotes cell survival in tissue culture. J Virol 75:3636-46.

17. Phelan D, Barrozo ER, Bloom DC. 2017. HSV1 latent transcription and non-coding RNA: A critical retrospective. J of Neuroimmunology 308:65-101.

18. Jones C. 2014. Reactivation from latency by alpha-herpesvirinae submfamily members: a stressful situation. Current Topics in Virology 12:99-118.

19. Glaser R, Kiecolt-Glaser JK, Speicher CE, Holliday JE. 1985. Stress, loneliness, and changes in herpesvirus latency. Journal of Behavioral Medicine 8:249-260.

20. Padgett DA, Sherida JF, Dorne J, Berntson GG, Candelora J, Glaser J. 1998. Social stress and the reactivation of latent herpes simplex virus type 1. Proc Natl Acad Sci U S A 95:7231-7235.

21. Jones C. 2016. Latency of Bovine Herpesvirus 1 (BoHV-1) in Sensory Neurons. In Omgradi J (ed), Herpesviridae. In Tech, doi:10.5772/63750.

22. Du T, Zhou G, Roizman B. 2012. Induction of apoptosis accelerates reactivation from latent HSV-1 in ganglionic organ cultures and replication in cell cultures. Proc Natl Acad Sci U S A 109:14616-14621.

23. Harrison K, Zhu L, Thunuguntla P, Jones C. 2019. Antagonizing the glucocorticoid receptor impairs explant-induced reactivation in mice latently infected with herpes simplex virus $1 \mathrm{~J}$ Virol 93:e00418-19.

24. Kook I, Henley C, Meyer F, Hoffmann F, Jones C. 2015. Bovine herpesvirus 1 productive infection and the immediate early transcription unit 1 are stimulated by the synthetic corticosteroid dexamethasone. Virology 484:377-385.

25. El-Mayet FS, Sawant L, Thungunutla P, Jones C. 2017. Combinatorial effects of the glucocorticoid receptor and Krüppel-like transcription factor 15 on bovine herpesvirus 1 transcription and productive infection. J Virol 91:91:e00904-17.

26. Erlandsson AC, Bladh L-C, Stierna, Yucel-Lindberg P, Hammersten O, Modeer T, Harmenberg T, Wikstrom AC. 2002. Herpes simplex virus type 1 infection and glucocorticoid treatment regulate viral yield, glucocorticoid receptor and NF-kB levels. Journal of Endocrinology 175:165-176.

27. Ostler J, Harrison KS, Schroeder K, Thunuguntla P, Jones C. 2019. The glucocorticoid receptor (GR) stimulates Herpes Simplex Virus 1 productive infection, in part because the infected cell protein 0 (ICP0) promoter is cooperatively transactivated by the GR and Krüppel-like transcription factor 15. J of Virol 93:e02063-18.

28. Oakley RH, Cidlowski AC. 2013. The biology of the glucocorticoid receptor: New signaling mechanisms in health and disease. J Allergy Clin Immunol 132:1033-1044. 
29. Workman A, Eudy J, Smith L, Frizzo da Silva L, Sinani D, Bricker H, Cook E, Doster A, Jones C. 2012. Cellular transcription factors induced in trigeminal ganglia during dexamethasone-induced reactivation from latency stimulate bovine herpesvirus 1 productive infection and certain viral promoters. J Virol 86:2459-2473.

30. McDonnell BB, Yang VW. 2010. Mammalian Kruppel-like factors in health and diseases. Physiol Rev 90:1337-1381.

31. Sinani D, Cordes E, Workman E, Thunuguntia P, Jones C. 2013. Stress induced cellular transcription factors expressed in trigeminal ganglionic neurons stimulate the herpes simplex virus type 1 (HSV-1) infected cell protein 0 (ICP0) promoter. J Virol 87:1183-1192.

32. Mallipattu SK, Guo Y, Revelo MP, Roa-Peña L, Miller T, Ling J, Shankland SJ, Bialkowska AB, Ly V, Estrada C, Jain MK, Lu Y, Ma'ayan A, Mehrotra A, Yacoub R, Nord EP, Woroniecki RP, Yang VW, He JC. 2017. Krüppel-Like Factor 15 Mediates Glucocorticoid-Induced Restoration of Podocyte Differentiation Markers. J Am Soc Nephrol 28:166-184.

33. Russell W. 1962. A Sensitive and Precise Plaque Assay for Herpes Virus. Nature 195:1028-1029.

34. Tremblay R, Sikorska M, Sandhu JK, Lanthier P, Ribecco-Lutkiewicz M, Bani-Yaghoub M. 2010. Differentiation of mouse Neuro-2A cells into dopamine neurons. J Neuroscience Methods 186:60-67.

35. Thunuguntla P, El-mayet FS, Jones C. 2017. Bovine herpesvirus 1 can efficiently infect the human (SH-SY5Y) but not the mouse neuroblastoma cell line (Neuro-2A). Virus Res 232:1-5.

36. Boutell C, Everret RD. 2013. Regulation of alphaherpesvirus infections by the ICP0 family of proteins. J Gen Virol 94:465-481.

37. Kaczynski J, Cook T, Urrutia R. 2003. Sp1- and Kruppel-like transcription factors. . Genome Biology 4:206.1-206.8.

38. Bieker JJ. 2001. Kruppel-like factors: three fingers in many pies. J Biol Chem 276:34355-34358.

39. Jones KA, Tjian R. 1985. Sp1 binds to promoter sequences and activates herpes simples virus 'immediate-early' gene transcription in vitro. Nature 317:179-182.

40. Black AR, Black JD, Azizkhan-Clifford J. 2001. Sp1 and Kruppel-like transcription factor family of transcription factors in cell growth and cancer. J Cell Physiol 188:143-160.

41. Mangan S, Alon U. 2003. Structure and function of the feed-forward loop network motif. Proc Natl Acad Sci U S A 100:1198011985.

42. Sasse S, Zuo Z, Kadiyala V, Zhang L, Pufall MA, Jain MK, Phang TL, Stormo GD, Gerber AN. 2015. Response element composition governs correlations between binding site affinity and transcription in glucocorticoid receptor feed-forward loops. J Biol Chem 290:19756-19769.

43. Sasse S, Mailoux CM, Barczak AJ, Wang Q, Altonsy MO, Jain MK, Haldar SM, Gerber AN. 2013. The glucocorticoid receptor and KLF15 regulate gene expression dynamics and integrate signals through feed-forward circuitry. Molec Cell Biol 33:21042115.

44. Inman M, Lovato L, Doster A, Jones C. 2002. A mutation in the latency-related gene of bovine herpesvirus 1 disrupts the latency reactivation cycle in calves. J Virol 76:6771-9.

45. Jones C, Newby TJ, Holt T, Doster A, Stone M, Ciacci-Zanella J, Webster CJ, Jackwood MW. 2000. Analysis of latency in cattle after inoculation with a temperature sensitive mutant of bovine herpesvirus 1 (RLB106). Vaccine 18:3185-95.

46. Winkler MT, Doster A, Sur JH, Jones C. 2002. Analysis of bovine trigeminal ganglia following infection with bovine herpesvirus 1. Vet Microbiol 86:139-55.

47. Winkler MT, Doster A, Jones C. 2000. Persistence and reactivation of bovine herpesvirus 1 in the tonsil of latently infected calves. J Virol 74:5337-5346.

48. Frizzo da Silva L, Kook I, Doster A, Jones C. 2013. Bovine herpesvirus 1 regulatory proteins, bICP0 and VP16, are readily detected in trigeminal ganglionic neurons expressing the glucocorticoid receptor during the early stages of reactivation from latency. J Virol 87:11214-11222.

49. Kook I, Doster A, Jones C. 2015. Bovine herpesvirus 1 regulatory proteins are detected in trigeminal ganglionic neurons during the early stages of stress-induced escape from latency. J Neurovirology 21:585-591.

50. Sasse S, Mailoux CM, Barczak AJ, Wang Q, Altonsy MO, Jain MK,. Haldar SM, Gerber AN. 2913. The glucocorticoid receptor and KLF15 regulate gene expression dynamics and integrate signals through feed-forward circuitry. Molec Cell Biol 33:21042115.

51. Ostler JB. Thunungutla P, Hendrickson BY, Jones C. 2021. Transactivation of HSV-1 infected cell protein 4 (ICP4) enhancer by glucocorticoid receptor and stress-induced transcription factors requires overlapping Krüppel like transcription factor 4/Sp1 binding sites. J Virol 95: e01776-20.

52. El-mayet F, L. Sawant, P. Thunuguntla, J. Zhao, and C. Jones. 2021. Two pioneer transcription factors, Krüppel-like transcription factor 4 and glucocorticoid receptor, cooperatively transactivate the bovine herpesvirus 1 ICP0 early promoter and stimulate productive infection. J Virol 94:e01670-19.

53. Everett RD. 2000. ICP0, a regulator of herpes simplex virus during lytic and latent infection. Bioessays 22:761-70.

54. Cliffe A, Knipe D. 2008. Herpes Simplex Virus ICP0 Promotes both Histone Removal and Acetylation on Viral DNA during Lytic Infection. J Virol 82:12030-12038.

55. Poon AP, Gu H, Roizman B. 2006. ICP0 and the US3 protein kinase of herpes simplex virus 1 independently block histone deacetylation to enable gene expression. Proc Natl Acad Sci U S A 103:9993-9998.

56. Zhang Y, Jones C. 2001. The bovine herpesvirus 1 immediate-early protein (bICP0) associates with histone deacetylase 1 to activate transcription. J Virol 75:9571-9578. 
57. Zhang Y, Jiang Y, Zhou J, and C. Jones. 2006. The bovine herpes virus 1 (BHV-1) immediate early protein (bICP0) interacts with the histone acetyltransferase p300, and these interactions correlate with stimulation of gC promoter activity. J Gen Virol 87:18431851.

58. Boutell C, Sadis S, Everett RD. 2002. Herpes simplex virus type 1 immediate-early protein ICP0 and its isolated RING finger domain act as ubiquitin E3 ligases in vitro. J Virol 76:841-850.

59. Boutell C, Everett RD. 2003. The herpes simplex virus type 1 (HSV-1) regulatory protein ICP0 interacts with an ubiquitinates p53. J Biol Chem 278:36596-36602.

60. Diao L, Qiao W, Chen Q, Wang C, Geng Y. 2005. bICP0 and its RING domain act as ubiquitin E3 ligases in vitro. Chinese Science Bulletin 50:636-640.

61. Parkinson J, Everett RD. 2001. Alphaherpesvirus proteins related to herpes simplex virus type 1 ICP0 induce the formation of colocalizing, conjugated ubiquitin. J Virol 75:5357-62.

62. Van Sant C, Hagglund R, Lopez P, Roizman B. 2001. The infected cell protein 0 of herpes simplex virus 1 dynamically interacts with proteasomes, binds and activates the cdc34 E2 ubiquitin- conjugating enzyme, and possesses in vitro E3 ubiquitin ligase activity. Proc Natl Acad Sci U S A 98:8815-20.

63. Uchida S, Tanaka Y, Ito H, Saitoh-Ohara F, Inazawa J, Yokoyama KK, Sasaki S, Marumo F. 2000. Transcriptional regulation of the CLC-K1 by myc-associated zinc finger protein, a novel zinc finger repressor. Molec Cell Biol 20:7319-7331.

64. Takeda K, Yahagi N, Aita Y, Murayama Y, Sawada Y, Piao X, Toya N, Oya Y, Shikama A, Takarada A, Masuda Y, Nishi M, Kuobota M, Izumida Y, Yamamoto T, Sekiya M, Matsuzaka T, Nakagawa Y, Urayama O, Kawakami Y, Iizuka Y, Gotoda T, Itaka K, Kataoka K, Nagai R, Kadowaki T, Yamada N, Lin Y, Jain MK, Shimano. H 2016. KLF15 enables switching between lipogenesis aand gluconeogenesis during fasting. Cell Reports 16:2373-2386.

65. Du X, Rosenfield RL, Qin K. KLF15 is a transcriptional regulator of the human 17beta-hydroxysteroid dehydrogenase type 5 gene. A potential link between regulation of testosterone production and fat stores in women. J Clin Endocrinol Metab 94:25942601.

66. Otteson DC, Lai H, Liu Y, Zack DJ. 2005. Zinc-finger domains of the transcriptional repressor KLF15 binds multiple sites in rhodopsin and IRBP promoters including the CRS-1 and G-rich elements. BMC Molecular Biology 6:doi:10.1186/1471-2199-615. 